\title{
Utilização do óleo de alho e da amoxilina, metronidazol e omeprazol no controle de Helicobacter spp. em cães
}

\author{
[Use of garlic oil and amoxicillin, metronidazole, and omeprazol in the control of Helicobacter spp. in dogs] \\ M.C. Costa ${ }^{1}$, P.R.S. Costa ${ }^{2}$, J.C.P. Silva ${ }^{2}$, R.E.N. Maia ${ }^{1}$, J.C.L. Moreira ${ }^{2}$, R.M. Carvalho ${ }^{3}$ \\ ${ }^{1}$ Médico veterinário autônomo \\ ${ }^{2}$ Departamento de Veterinária - UFV - Viçosa, MG \\ ${ }^{3}$ Farmacêutica bioquímica autônoma
}

\begin{abstract}
RESUMO
Avaliaram-se a eficácia do óleo de alho e da terapia tripla (amoxicilina, metronidazol e omeprazol) no tratamento de 21 cães infectados por Helicobacter spp., que apresentavam alterações histológicas nas biopsias endoscópicas da mucosa gástrica e reação positiva ao teste de urease. Os animais foram distribuídos, aleatoriamente, em três grupos de sete cães, os quais receberam os seguintes tratamentos: grupo 1 - cápsulas vazias; grupo 2 - 500mg de óleo de alho em cápsulas, diariamente, por um período de 30 dias; grupo 3 - amoxicilina, metronidazol e omeprazol, respectivamente, nas doses de $20 \mathrm{mg} / \mathrm{kg}$ a cada 12 horas, $25 \mathrm{mg} / \mathrm{kg}$ e $20 \mathrm{mg} / \mathrm{kg}$ a cada 24 horas, durante 15 dias. Ao final dos tratamentos, os cães foram submetidos à endoscopia com realização de biopsias da mucosa gástrica. $\mathrm{O}$ tratamento com amoxicilina, metronidazol e omeprazol resultou em erradicação de Helicobacter spp. tanto na região fúndica quanto na pilórica. No grupo 2, houve redução da degeneração glandular na região fúndica em dois animais e em outros dois na pilórica. O tratamento com óleo de alho não foi eficaz em erradicar Helicobacter spp., apenas reduziu a sua colonização em quatro dos animais tratados.
\end{abstract}

Palavras-chave: cão, Helicobacter spp., óleo de alho, amoxicilina, metronidazol, omeprazol

\begin{abstract}
The efficacy of garlic oil and triple therapy (amoxicillin, metronidazole, and omeprazol) were evaluated in the treatment of 21 dogs infected by Helicobacter spp., which presented histological alterations of the gastric mucosa according to endoscopic biopsies and positive reaction to urease test. The animals were randomly distributed into three groups of seven dogs each, and received the following treatment, group 1 - empty capsules; group 2 - 500mg of garlic oil capsules daily for a period of 30 days; and group 3 amoxicillin, metronidazole, and omeprazol, in doses of 20mg/kg every 12 hours, $25 \mathrm{mg} / \mathrm{kg}$ and $20 \mathrm{mg} / \mathrm{kg}$ every 24 hours, respectively, for 15 days. By the end of the treatment, the dogs were subjected to new endoscopic procedure with gastric mucosa biopsies. Treatment with amoxicillin, metronidazole, and omeprazol resulted in eradication of Helicobacter spp. both in fundic and pyloric regions. The garlic oil treatment reduced glandular degeneration in the fundic region in two animals and in the pylorus region in two other animals, and it was inefficient in eradicating Helicobacter spp. in dogs as it only reduced the colonization in some of the treated animals.
\end{abstract}

Keywords: dog, Helicobacter spp., garlic oil, amoxicillin, metronidazole, omeprazol

\section{INTRODUÇÃO}

Um novo fator patogênico relacionado com a gastrite crônica em atividade foi verificado, pela primeira vez, por Warren e Marshal (1983) ao isolarem bactérias espiraladas em biopsias gástricas de pacientes humanos com processo inflamatório. Essa bactéria, Helicobacter pylori, é reconhecida como a mais importante causa de gastrite crônica em humanos e é também

Recebido em 23 de novembro de 2007

Aceito em 4 de fevereiro de 2009

E-mail: mqmanu@hotmail.com 
implicada como determinante etiológico de úlceras pépticas, sobretudo as duodenais (Canizares et al., 2002). Helicobacter spp. são bactérias Gram- negativo com relativa capacidade de se multiplicar no estômago, graças à produção e à atividade da enzima urease (Belli et al., 2003).

Em medicina veterinária, Helicobacter spp. têm sido relacionados com inflamação da mucosa gastrintestinal em diferentes espécies, embora a correlação entre colonização e manifestações clínicas ainda não esteja bem esclarecida (Belli et al., 2003; Baillon e Marshal-Jones, 2004).

Segundo Jenkins e Bassett (1997) e Neiger e Simpson (2000), há relatos de infecção gástrica por espécies de Helicobacter em gatos, cães, ratos, suínos, bovinos, raposas, felinos selvagens e primatas não humanos.

Cães e gatos são infectados por diferentes espécies de Helicobacter, incluindo: $H$. felis, $H$. heilmannii, $H$. bizzozeronii, $H$. bitis, $H$. salomonis e $H$. rappini. A prevalência de animais portadores situa-se entre 70 e 100\% (Happonen et al., 2000; Baillon e Marshal-Jones, 2004).

O potencial zoonótico dessas bactérias justifica o crescente e renovado interesse da medicina veterinária, em especial, no estabelecimento da incidência e no significado clínico em animais domésticos, sobretudo em cães e gatos, já que microrganismos com morfologias semelhantes a H. pylori foram observados no estômago de animais com gastrite (Handt et al., 1995).

Martin e Ernst (2003) relataram que a erradicação de $H$. pylori pode ser difícil com o uso da antibioticoterapia convencional, requerendo a combinação de dois antibióticos associados a um inibidor da bomba de prótons, para diminuir a produção de ácido clorídrico presente no suco gástrico. Entretanto, segundo O'Gara et al. (2000), a resistência da bactéria aos antibióticos é um dos principais problemas encontrados. Dessa forma, vários tratamentos alternativos têm sido propostos, tais como a utilização de óleo de alho, cuja atividade anti- $H$. pylori foi demonstrada por meio de estudos in vitro. (Cellini et al., 1996; Sivam et al., 1997; Canizares et al., 2004).
O objetivo deste estudo foi avaliar a terapia tripla (amoxicilina, metronidazol e omeprazol) e verificar a eficácia do óleo de alho no tratamento da infecção por Helicobacter spp. em cães.

\section{MATERIAL E MÉTODOS}

Foram utilizados 21 cães adultos, clinicamente sadios, sem raças definidas, oito machos e 13 fêmeas, com pesos entre seis e $25 \mathrm{~kg}$.

Após jejum sólido de 12 horas e hídrico de duas horas, os animais receberam, como medicação pré-anestésica, acepromazina $1 \%$ na dose de $0,1 \mathrm{mg} / \mathrm{kg}$ pela via intravenosa (IV). Cerca de 10 minutos depois, a anestesia geral foi induzida com tiopental sódico ${ }^{2}$ na dose de $12,5 \mathrm{mg} / \mathrm{kg} / \mathrm{IV}$. Após atingir o plano anestésico, foi iniciada a endoscopia e posteriormente as biopsias. Os animais foram posicionados em decúbito lateral esquerdo, o que possibilitou a introdução de um gastroscópio, modelo Olympus CV-1, com 9,5mm de diâmetro.

Procedeu-se aos exames da integridade da mucosa observando-se coloração, presença de lesões pré-ulcerativas e/ou ulcerativas, alterações circulatórias, presença de parasitas e outras alterações de superfície. Após a endoscopia, foram coletados fragmentos da mucosa gástrica, obedecendo a um mapeamento previamente realizado e que correspondia às regiões do corpo e do antrogástrico, para a realização de exames citológicos, histológico e do teste de urease. Após esses procedimentos, os animais retornaram para as suas baias, onde permaneceram até o final do tratamento, quando foram realizados novos exames endoscópicos e novas biopsias.

Para o teste rápido de urease dos fragmentos de mucosa colhidos na região do corpo gástrico, utilizou-se $k_{i t}{ }^{l}$ comercial, para detectação de $H$. pylori, que continha ureia e vermelho fenol. $\mathrm{O}$ teste foi considerado positivo quando a solução adquiria tonalidade rosa, indicando presença da bactéria devido à liberação de amônia. A reação foi considerada fortemente positiva nos casos em que a viragem do meio ocorreu entre uma e três horas, ou fracamente positiva nas amostras em que a reação ocorreu após quatro horas.

${ }^{1}$ Iris d'água Ltda - Belo Horizonte, Brasil. 
Os fragmentos de mucosa gástrica foram fixados em formol neutro tamponado a $10 \%$, por 24 horas, desidratados em concentrações crescentes de álcool, clareados em xilol e incluídos em parafina, seguindo-se a técnica preconizada para utilização na rotina em histologia.

Os cortes com espessura de $4 \mu \mathrm{m}$ foram corados e analisados em microscopia de luz, sendo os resultados expressos em diferentes graus, utilizando-se para tal os seguintes parâmetros: infiltração inflamatória, degenerações glandulares, número de aglomerados linfoides e concentração de bactérias espiraladas, por campo microscópico (400X). A coloração pela carbolfucsina foi utilizada para identificar bactérias espiraladas do gênero Helicobacter e quantificar o grau de colonização.

A coloração pela hematoxilina-eosina foi usada para se realizar uma descrição histológica adequada, incluindo a identificação de eventuais alterações inflamatórias, a natureza e o número de infiltrados celulares, a degeneração glandular e a presença de aglomerados linfoides na mucosa gástrica. O grau de gastrite foi estimado com base no número de infiltrados de linfócitos, de plasmócitos ou de polimorfonucleares na lâmina própria.
Os animais utilizados foram distribuídos, aleatoriamente, em três grupos com sete animais cada, que receberam os seguintes tratamentos: grupo-controle - cápsulas vazias; grupo 2 $500 \mathrm{mg}$ de óleo de alho em cápsulas, diariamente, por um período de 30 dias; grupo 3 amoxicilina $^{2}$, metronidazol ${ }^{3}$ e omeprazol $^{4}$, respectivamente, nas doses de $20 \mathrm{mg} / \mathrm{kg}$ a cada 12 horas, $25 \mathrm{mg} / \mathrm{kg}$ e $20 \mathrm{mg} / \mathrm{kg}$ a cada 24 horas, durante 15 dias consecutivos. Após este período, os animais foram submetidos a um novo exame endoscópico e à colheita de fragmentos das regiões contralateral da mucosa fúndica e antral do estômago.

Para análise histológica qualitativa, na qual foram atribuídos escores, consideraram-se a densidade de bactérias espiraladas por campo (400x), a presença de células inflamatórias, o número de agregados linfoides e a existência de alterações degenerativas glandulares (Happonen et al., 1998; Iimuro et al., 2002), conforme Tab. 1.

Para a análise estatística, utilizou-se o sistema SAEG 4.0, compararam-se as médias por meio do teste Wilcoxon $(\mathrm{P}<5 \%)$ e estimou-se o coeficiente de correlação de Pearson.

Tabela 1. Escore para avaliação histológica da mucosa gástrica de cães

\begin{tabular}{|c|c|c|c|c|}
\hline Escore & $\begin{array}{c}\text { Bactérias espiraladas } \\
\text { (densidade) }\end{array}$ & $\begin{array}{l}\text { Infiltrado inflamatório } \\
\text { (intensidade) }\end{array}$ & $\begin{array}{c}\text { Agregados } \\
\text { linfoides (n) }\end{array}$ & $\begin{array}{l}\text { Degeneração } \\
\text { glandular }\end{array}$ \\
\hline 0 & ausente & normal & ausente & ausente \\
\hline 1 & leve & leve & 1 & $\begin{array}{l}<20 \% \text { de glândulas } \\
\text { com degeneração }\end{array}$ \\
\hline 2 & moderada & moderada & 2 & $\begin{array}{l}20 \text { a } 50 \% \text { de glândulas } \\
\text { com degeneração }\end{array}$ \\
\hline 3 & severa & severa & $\geq 3$ & $\begin{array}{l}>50 \% \text { de glândulas } \\
\text { com degeneração }\end{array}$ \\
\hline
\end{tabular}

\section{RESULTADOS E DISCUSSÃO}

Os resultados que expressam todos os parâmetros analisados encontram-se nas Tab. 2,3 e 4 . O tratamento que apresentou melhor resultado foi o da terapia tripla, que, ao final do procedimento, resultou em erradicação de Helicobacter spp.

\footnotetext{
${ }^{2}$ Univet S.A. - São Paulo, Brasil.

${ }^{3}$ Cristália Prod. Quim. Farm. Ltda. - Itapira, Brasil.

${ }^{4}$ Rambaxy S.A. - São Paulo Brasil.
} 
Utilização do óleo de alho...

Tabela 2. Escore do grau de inflamação e do grau de colonização de Helicobacter spp. em cães do grupocontrole, antes e após o tratamento ${ }^{1}$

\begin{tabular}{|c|c|c|c|c|c|c|c|c|c|c|c|c|c|c|c|c|}
\hline \multirow{4}{*}{ Animal } & \multicolumn{12}{|c|}{ Grau de inflamação } & \multicolumn{4}{|c|}{ Grau de colonização } \\
\hline & \multicolumn{6}{|c|}{ No corpo } & \multicolumn{6}{|c|}{ No antro } & \multicolumn{2}{|c|}{ No corpo } & \multicolumn{2}{|c|}{ No antro } \\
\hline & \multicolumn{3}{|c|}{ Antes } & \multicolumn{3}{|c|}{ Depois } & \multicolumn{3}{|c|}{ Antes } & \multicolumn{3}{|c|}{ Depois } & Antes & Depois & Antes & Depois \\
\hline & EI & $\mathrm{AL}$ & DG & EI & $\mathrm{AL}$ & DG & EI & $\mathrm{AL}$ & DG & EI & $\mathrm{AL}$ & DG & $\mathrm{HE}$ & $\mathrm{HE}$ & $\mathrm{HE}$ & $\mathrm{HE}$ \\
\hline CA & 0 & 0 & 1 & 1 & 0 & 0 & 1 & 0 & 1 & 2 & 0 & 0 & 3 & 2 & 3 & 3 \\
\hline $\mathrm{CB}$ & 1 & 0 & 0 & 1 & 0 & 1 & 1 & 0 & 0 & 1 & 0 & 1 & 3 & 3 & 3 & 3 \\
\hline $\mathrm{CC}$ & 1 & 0 & 0 & 1 & 1 & 1 & 1 & 0 & 0 & 1 & 0 & 1 & 2 & 2 & 3 & 3 \\
\hline $\mathrm{CD}$ & 2 & 2 & 1 & 2 & 2 & 1 & 2 & 0 & 1 & 2 & 0 & 1 & 3 & 3 & 2 & 3 \\
\hline $\mathrm{CE}$ & 0 & 0 & 1 & 0 & 0 & 0 & 0 & 0 & 1 & 0 & 0 & 0 & 2 & 2 & 3 & 3 \\
\hline $\mathrm{CF}$ & 2 & 0 & 1 & 2 & 3 & 1 & 2 & 0 & 1 & 1 & 0 & 1 & 2 & 2 & 2 & 2 \\
\hline CG & 1 & 0 & 0 & 0 & 1 & 1 & 1 & 3 & 0 & 0 & 0 & 0 & 2 & 3 & 3 & 3 \\
\hline
\end{tabular}

${ }^{\mathrm{T}}$ Cápsulas de placebo.

EI: escore de infiltrado inflamatório; AL: aglomerado linfoide; DG: degeneração glandular; EH: colonização por Helicobacter spp.

Tabela 3. Escore do grau de inflamação e do grau de colonização de Helicobacter spp. no grupo 2, antes e após o tratamento ${ }^{1}$

\begin{tabular}{|c|c|c|c|c|c|c|c|c|c|c|c|c|c|c|c|c|}
\hline \multirow{4}{*}{ Animal } & \multicolumn{12}{|c|}{ Grau de inflamação } & \multicolumn{4}{|c|}{ Grau de colonização } \\
\hline & \multicolumn{6}{|c|}{ No corpo } & \multicolumn{6}{|c|}{ No antro } & \multicolumn{2}{|c|}{ No corpo } & \multicolumn{2}{|c|}{ No antro } \\
\hline & \multicolumn{3}{|c|}{ Antes } & \multicolumn{3}{|c|}{ Depois } & \multicolumn{3}{|c|}{ Antes } & \multicolumn{3}{|c|}{ Depois } & Antes & Depois & Antes & Depois \\
\hline & EI & $\mathrm{AL}$ & DG & EI & $\mathrm{AL}$ & DG & EI & $\mathrm{AL}$ & DG & EI & $\mathrm{AL}$ & DG & HE & $\mathrm{HE}$ & $\mathrm{HE}$ & $\mathrm{HE}$ \\
\hline AA & 1 & 0 & 0 & 1 & 0 & 0 & 0 & 0 & 0 & 1 & 0 & 0 & 3 & 2 & 3 & 3 \\
\hline $\mathrm{AB}$ & 0 & 2 & 0 & 0 & 0 & 0 & 0 & 0 & 0 & 0 & 0 & 0 & 2 & 1 & 2 & 1 \\
\hline $\mathrm{AC}$ & 2 & 1 & 2 & 1 & 0 & 0 & 1 & 0 & 0 & 1 & 0 & 0 & 3 & 3 & 3 & 2 \\
\hline $\mathrm{AD}$ & 2 & 0 & 2 & 0 & 0 & 0 & 0 & 0 & 0 & 0 & 0 & 0 & 3 & 3 & 3 & 3 \\
\hline $\mathrm{AE}$ & 1 & 0 & 1 & 0 & 0 & 0 & 1 & 0 & 1 & 0 & 0 & 0 & 2 & 1 & 2 & 0 \\
\hline $\mathrm{AF}$ & 0 & 0 & 1 & 0 & 0 & 0 & 0 & 0 & 1 & 0 & 0 & 0 & 3 & 2 & 3 & 3 \\
\hline AG & 1 & 0 & 0 & 0 & 0 & 0 & 0 & 0 & 0 & 0 & 0 & 0 & 3 & 2 & 3 & 2 \\
\hline
\end{tabular}

${ }^{1} 500 \mathrm{mg}$ de óleo de alho em cápsulas diariamente, durante 30 dias.

EI: escore de infiltrado inflamatório; AL: aglomerado linfoide; DG: degeneração glandular; EH: colonização por Helicobacter spp.

Tabela 4. Escore do grau de inflamação e do grau de colonização de Helicobacter spp. no grupo 3, antes e após o tratamento 1

\begin{tabular}{|c|c|c|c|c|c|c|c|c|c|c|c|c|c|c|c|c|}
\hline \multirow{4}{*}{ Animal } & \multicolumn{12}{|c|}{ Grau de inflamação } & \multicolumn{4}{|c|}{ Grau de colonização } \\
\hline & \multicolumn{6}{|c|}{ No corpo } & \multicolumn{6}{|c|}{ No antro } & \multicolumn{2}{|c|}{ No corpo } & \multicolumn{2}{|c|}{ No antro } \\
\hline & \multicolumn{3}{|c|}{ Antes } & \multicolumn{3}{|c|}{ Depois } & \multicolumn{3}{|c|}{ Antes } & \multicolumn{3}{|c|}{ Depois } & Antes & Depois & Antes & Depois \\
\hline & $\mathrm{EI}$ & $\mathrm{AL}$ & $\mathrm{DG}$ & EI & $\mathrm{AL}$ & DG & EI & $\mathrm{AL}$ & $\overline{\mathrm{DG}}$ & EI & $\mathrm{AL}$ & DG & $\mathrm{HE}$ & $\mathrm{HE}$ & $\mathrm{HE}$ & $\mathrm{HE}$ \\
\hline TA & 1 & 0 & 1 & 1 & 0 & 1 & 1 & 0 & 1 & 1 & 0 & 1 & 3 & 0 & 3 & 0 \\
\hline TB & 1 & 0 & 1 & 1 & 0 & 1 & 1 & 0 & 1 & 1 & 0 & 1 & 1 & 0 & 2 & 0 \\
\hline $\mathrm{TC}$ & 1 & 0 & 1 & 1 & 1 & 1 & 2 & 0 & 1 & 1 & 0 & 1 & 3 & 0 & 3 & 0 \\
\hline TD & 1 & 0 & 1 & 1 & 0 & 1 & 1 & 0 & 1 & 2 & 0 & 1 & 3 & 0 & 2 & 0 \\
\hline $\mathrm{TE}$ & 0 & 0 & 1 & 1 & 0 & 1 & 1 & 0 & 1 & 2 & 0 & 1 & 3 & 0 & 3 & 0 \\
\hline $\mathrm{TF}$ & 1 & 0 & 1 & 1 & 0 & 1 & 2 & 0 & 1 & 1 & 0 & 1 & 2 & 0 & 2 & 0 \\
\hline TG & 1 & 0 & 2 & 0 & 0 & 1 & 2 & 2 & 2 & 1 & 0 & 1 & 3 & 0 & 3 & 0 \\
\hline
\end{tabular}

${ }^{1}$ Amoxicilina, metronidazol e omeprazol nas doses de $20 \mathrm{mg} / \mathrm{kg}$ a cada 12 horas, $25 \mathrm{mg} / \mathrm{kg}$ e $20 \mathrm{mg} / \mathrm{kg}$ a cada 24 horas, respectivamente.

EI: escore de infiltrado inflamatório; AL: aglomerado linfoide; DG: degeneração glandular; EH: colonização por Helicobacter spp.

A terapia tripla consiste na combinação de dois antibióticos e um inibidor da bomba de prótons e, devido à sua eficácia, tem o seu uso gradativamente ampliado no tratamento clínico de H. pylori, associado a outras doenças gastrintestinais (Iimuro et al., 2002). No entanto, a erradicação pela terapia tripla nem sempre é eficaz, e alguns efeitos colaterais dessas drogas 
têm sido reportados. Martin e Ernst (2003) relataram que a erradicação de $H$. pylori pode ser difícil com o uso da antibioticoterapia convencional e requer, para tal, a combinação de antibióticos, inibidores da bomba de prótons e preparados à base de bismuto. Similarmente, Simpson e Burrows (1997) enfatizaram que nenhum medicamento antimicrobiano, por si só, tem resultado em taxa adequada de erradicação dessas bactérias espiraladas. Desse modo, Flatland (2002) afirma que a resistência de $H$. pylori aos antibióticos constitui um sério problema na medicina humana e tem sido encontrada com a claritromicina, o metronidazol, a amoxicilina e a tetraciclina. Entretanto, combinações com novas terapias têm sido feitas com sucesso, utilizando-se o metronidazol, apesar de sua aparente resistência in vitro. Neste estudo, o protocolo da terapia tripla foi realizado com sucesso, utilizando-se os antibióticos amoxicilina e metronidazol, juntamente com o omeprazol.

Apesar de a resistência a esses antibióticos ter sido relatada por O'Gara et al. (2000), isso não foi observado neste experimento, pois os animais tiveram queda consistente da colonização e da densidade da bactéria comprovada tanto pelo teste de urease quanto pelo exame histológico (Fig. 1). Estes resultados diferem, pelo menos em parte, dos observados por Flatland (2002), que sugeriu que o tratamento pode apenas suprimir ligeiramente a infecção, porém não é capaz de eliminá-la.

Não se observou correlação entre a redução da densidade bacteriana e os outros parâmetros avaliados. Dessa forma, a densidade de bactérias não está diretamente correlacionada com o número de células inflamatórias, com agregados linfoides e com degeneração glandular, pois, mesmo havendo a erradicação de Helicobacter spp. não houve redução significativa no valor dessas variáveis (Fig. 2 e 3). No entanto, esses dados devem ser interpretados com cuidado, uma vez que as biopsias foram realizadas logo após o término do tratamento, isto é, foi curto o tempo estudado até a cessação do estímulo agressor, o que poderia não ser suficiente para provocar redução dos parâmetros citados. Simpson et al. (1999) afirmaram não haver relação entre o grau de inflamação e o grau de colonização por bactérias, resultado não confirmado por Vieira (2004).

Os animais tratados com o óleo de alho apresentaram redução significativa do infiltrado inflamatório, da degeneração glandular e da densidade de Helicobacter spp. na região do corpo gástrico. Ainda, houve redução significativa da degeneração glandular tanto da região fúndica quanto da região antral. Segundo Iimuro et al. (2002), o extrato de alho na dieta apresenta pouca ou nenhuma capacidade de erradicar $H$. pylori ou reduzir as lesões gástricas em gerbils depois que a lesão estiver estabelecida. Entretanto, ele pode prevenir a indução de gastrite por $H$. pylori.

Efeitos sinérgicos do extrato de alho com omeprazol in vitro sugerem que o seu uso, com o inibidor da bomba de prótons, pode ser indicado com sucesso (Cellini et al., 1996; O'Gara et al., 2000).

Há relatos de irritação gástrica, logo, a alicina, que é o princípio ativo do alho, deve ser usada com cautela em certos pacientes, sobretudo naqueles portadores de lesão da mucosa gástrica ou inflamação (O'Gara et al., 2000). Nos animais tratados com cápsulas de alho, não foi observado aumento no grau de inflamação ou lesão na mucosa gástrica e, sim, diminuição.

Graham et al. (1999) observaram efeitos colaterais como diarreia, flatulência, hálito e odor de alho pelo corpo nos pacientes tratados, contudo, na presente pesquisa, não foi verificado qualquer tipo de efeito colateral nos animais submetidos a esse tratamento.

A terapia com alho resultou em redução da densidade de bactérias espiraladas no estômago de cães, diferente do que foi constatado por Ohta et al. (1999), Sivam (2001) e Canizares et al. (2002), em estudos experimentais realizados in vitro.

Segundo O'Gara et al. (2000), na prática, o maior problema com a ingestão de preparados à base de alho, em tablete ou em cápsula, reside na inativação ou destruição da alicinase pelo $\mathrm{pH}$ gástrico, prevenindo a formação da alicina e diminuindo, assim, tanto a atividade sistêmica como a intragástrica do componente ativo. Mcnulty et al. (2001) asseguraram que a formação da alicinase ocorre quando se corta o dente de alho, ou se adiciona água ao pó de alho, e que sua atividade é inativada pelo suco gástrico.

Estudos realizados por Canizares et al. (2002), nos quais foram comparadas as capacidades inibitórias do extrato de alho e de alguns antibióticos usados no tratamento de infecções por $H$. pylori, sugeriram que o extrato de alho possui graus de inibição in vitro similar ao desses antibióticos. 

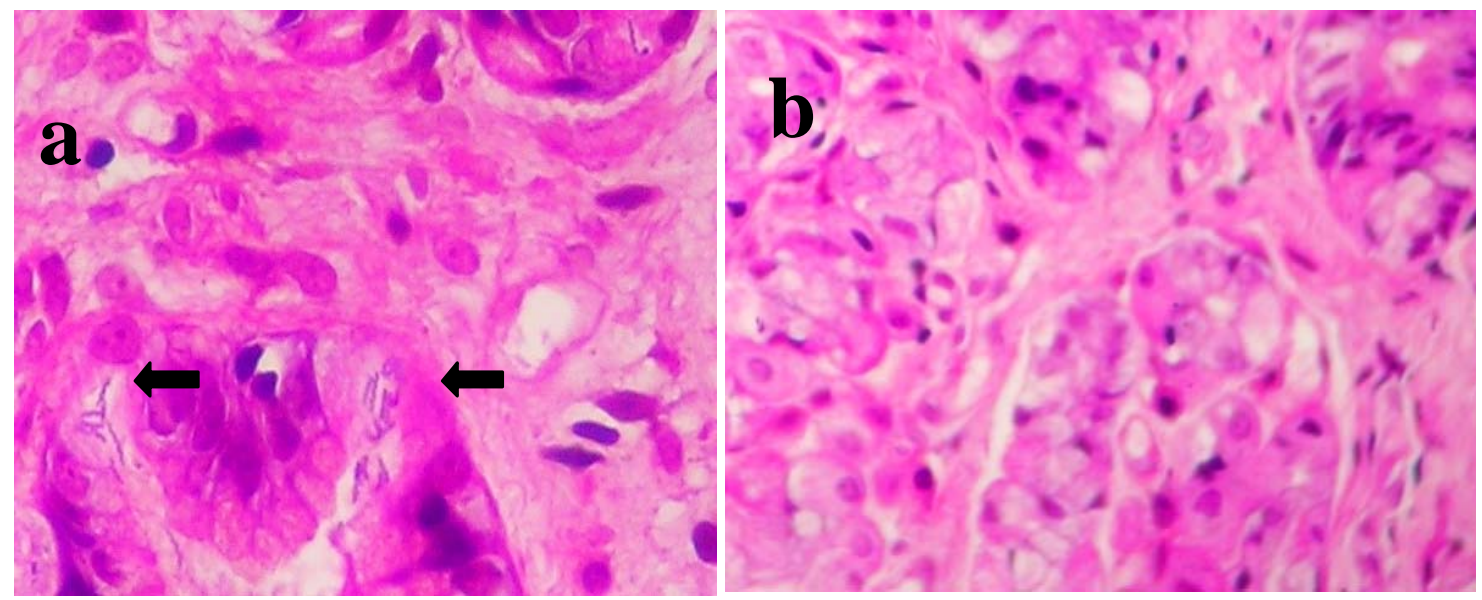

Figura 1. Corte histológico da mucosa gástrica antes (a $\leftarrow$ - escore 3 ) e após o tratamento com a terapia tripla $^{1}$ (b) no mesmo cão (Carbol-fucsina, 400X).

${ }^{1}$ Amoxicilina, metronidazol e omeprazol nas doses de $20 \mathrm{mg} / \mathrm{kg}$ a cada 12 horas, $25 \mathrm{mg} / \mathrm{kg}$ e $20 \mathrm{mg} / \mathrm{kg}$ a cada 24 horas, respectivamente.
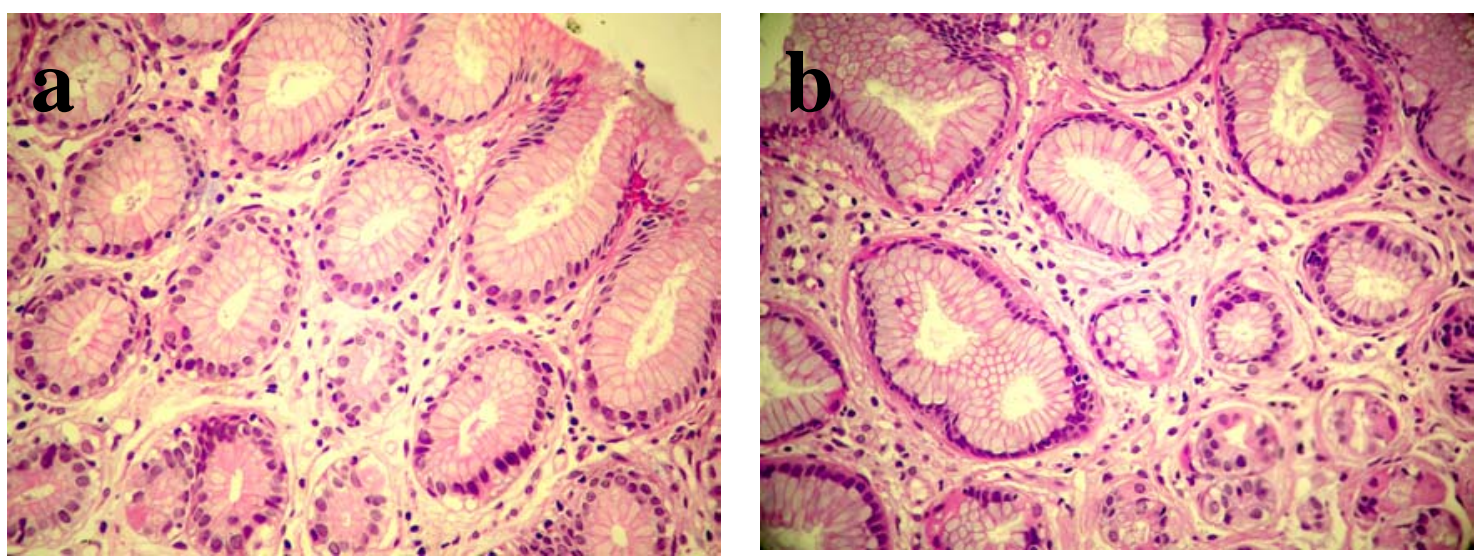

Figura 2. Corte histológico da mucosa gástrica com infiltrado inflamatório linfoplasmocitário (escore 1) antes (a) e após o tratamento (b) com a terapia tripla ${ }^{1}$ no mesmo cão (HE, 400X).

${ }^{1}$ Amoxicilina, metronidazol e omeprazol nas doses de $20 \mathrm{mg} / \mathrm{kg}$ a cada 12 horas, $25 \mathrm{mg} / \mathrm{kg}$ e $20 \mathrm{mg} / \mathrm{kg}$ a cada 24 horas, respectivamente.
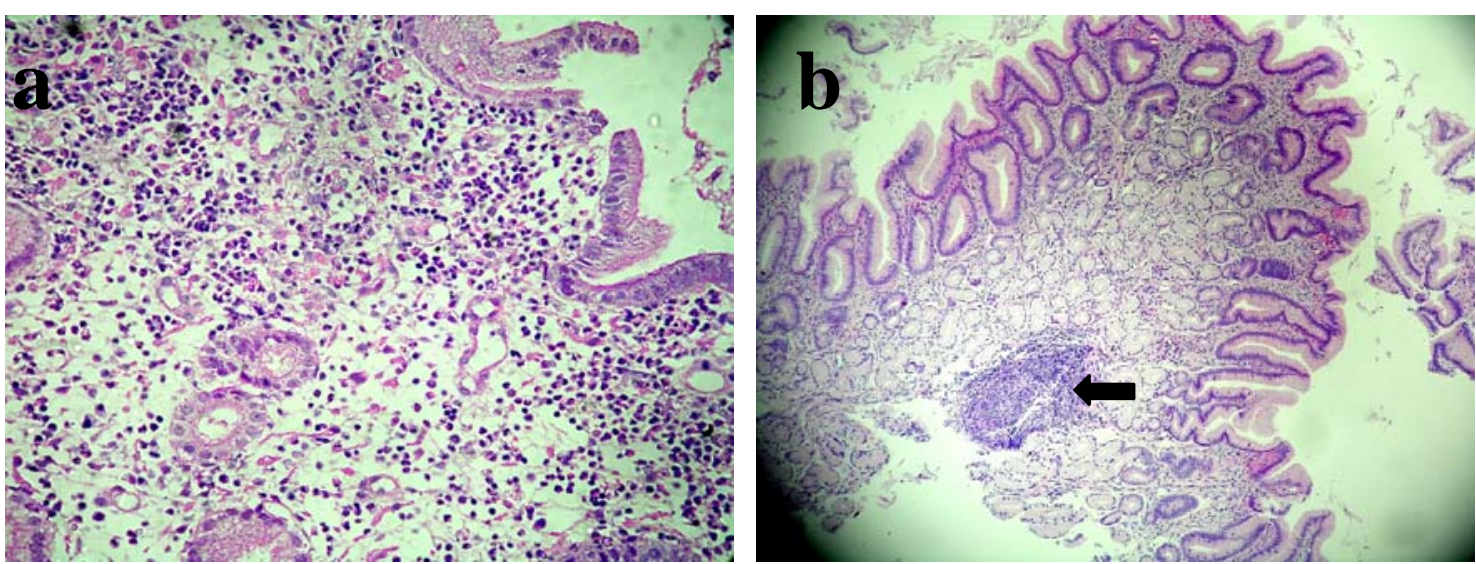

Figura 3. Corte histológico da mucosa gástrica com infiltrado inflamatório linfoplasmocitário (escore 2) (a) e presença de folículo linfoide $(\mathrm{b} \leftarrow$ ) no mesmo cão (HE, 50 e 100X). 


\section{CONCLUSÕES}

O uso da terapia tripla aplicada aos humanos para o tratamento de Helicobacter pylori mostrou-se efetivo no tratamento das bactérias gástricas espiraladas presentes nos cães. O tratamento com óleo de alho na dose utilizada foi eficaz em reduzir a degeneração glandular e não foi eficaz em erradicar Helicobacter spp. em cães.

\section{REFERÊNCIAS BIBLIOGRÁFICAS}

BAILLON, M.L.; MARSHALL-JONES, Z. Bactérias enteropatogênicas em cães e gatos. Waltham Focus Ed. Esp., p.12-18, 2004.

BELLI, C.B; FERNANDES, W.R.; SILVA, L.C.L.C. Teste de urease positivo em equino com úlcera gástrica - Helicobacter sp.? Arq. Inst. Biol. São Paulo, v.70, p.17-20, 2003.

CANIZARES, P.; GRACIA, I.; GÓMEZ, L.A. et al. Thermal degradation of allicin in garlic extracts and its implication on the inhibition of the in-vitro growth of Helicobacter pylori. Biotechnol. Prog., v.20, p.32-37, 2004

CANIZARES, P.; GRACIA, I.; GÓMEZ, L. A et al. Optimization of Allium sativum solvent extraction for the inhibition of in vitro growth of Helicobacter pylori. Biotechnol. Prog., v.18, p.1227-1232, 2002.

CELLINI, L.; DI CAMPLI, E.; MASSULI, $\mathrm{M}$ et al. Inhibition of Helicobacter pylori by garlic extract (Allium sativum). FEMS Immunol. Med. Microbiol., v.13, p.273-277, 1996.

FLATLAND, B. Helicobacter infection in humans and animals. Compend. Contin. Educ. Pract. Vet., v.24, p.688-698, 2002.

GRAHAM, D.Y.; ANDERSON, S.Y.; LANG, T. Garlic or jalapeno peppers for treatment of Helicobacter pylori infection. Am. J. Gastroenterol., v.94, p.1200-1202, 1999.

HANDT, L.K.; FOX, J.G; STALIS, I.H. et al. Characterization of feline Helicobacter pylori strains and associated gastritis in a colony of domestic cats. $J$. Clin. Microbiol., v.33, p.2280-2289, 1995.

HAPPONEN, I.; LINDEN, J.; SAARI, S. et al Detection and effects of helicobacters in healthy dogs and dogs with signs of gastritis. J. Am. Vet. Med. Assoc., v.213, p.1767-1774, 1998.

HAPPONEN, I.; LINDEN, J.; WESTERMARCK, Effect of the triple therapy on eradication of canine gastric helicobacters and gastric disease. J. Small Anim. Pract., v.41, p.1-6, 2000.
IIMURO, M.; SHIBATA, H.; KAWAMORI, T. et al. Suppressive effects of garlic extract on Helicobacter pylori-induced gastritis in Mongolian gerbils. Cancer Lett., v.187, p.61-68, 2002.

JENKINS, C.C.; BASSET, J.R. Helicobacter Infection. Compend. Contin. Educ. Pract. Vet., v.19, p.267-279, 1997.

MARTIN, K.W.; ERNST, E. Herbal medicines for treatment of bacterial infections: a review of controlled clinical trials. J. Antimicrob. Chemother., v.51, p.241-246, 2003.

MCNULTY, C.A.; WILSON, M.P.; HAVINGA, W. et al. A pilot study to determine the effectiveness of garlic oil capsules in the treatment of dyspeptic patients with Helicobacter pylori. Helicobacter, v.6, p.249-253, 2001.

NEIGER, R.; SIMPSON, K.W. Helicobacter infection in dogs and cats: facts and fictions. J. Vet. Intern. Med., v.14, p.125-133, 2000.

O'GARA, E.A.; HILL, D.J.; MASLIN, D.J. Activities of garlic oil, garlic powder, and their diallyl constituents against Helicobacter pylori. Appl. Environ. Microbiol., v.66, p.2269-2273, 2000.

OHTA, R.; YAMADA, N.; KANEKO, H. et al. In vitro inhibition of the growth of Helicobacter pylori by oil-macerated garlic constituents. Antimicrob. Agents Chemother., v.43, p.1811-1812, 1999.

SIMPSON, K.W.; BURROWS, C.F. Gastritis, Ulcers and Helicobacter spp. in humans, dogs and cats. Waltham Focus, v.7, p.2-6, 1997.

SIMPSON, K.W.; MCDONOUGH, P.L.; STRAUSSAYALI, D. et al. Helicobacter felis infection in dogs: effect on gastric structure and function. Vet. Pathol., v.36, p.237-248, 1999.

SIVAM, G.P. Protection against Helicobacter pylori and other bacterial infections by garlic. J. Nutr., v.131, p.1106S-1108S, 2001.

SIVAM, G.P.; LAMPE, J.W.; ULNESS, B. et al. J.D. Helicobacter pylori-In vitro susceptibility to garlic (Allium sativum) extract. Nutr. Câncer, v.27, p.118121, 1997.

VIEIRA, F.T. Frequência e distribuição de Helicobacter spp. na mucosa gástrica de cães. 2004. 54f. Dissertação (mestrado) - Universidade Federal de Viçosa, Viçosa, MG.

WARREN, J.; MARSHALL, B. Unidentified curved bacilii on gastric epithelium in active chronic gastritis. Lancet, v.1, p.1273, 1983. 